\title{
Fifty years in fusion and the way forward
}

\author{
J. Jacquinot \\ Cabinet of the French High Commissioner for Atomic Energy CEA, 91191 Gif-sur-Yvette, \\ France
}

Received 19 March 2009, accepted for publication 1 July 2009

Published 30 December 2009

Online at stacks.iop.org/NF/50/014001

\begin{abstract}
This particular 'Fusion Pioneers Memorial lecture' was given 50 years after the first historical FEC conference in 1958. It was a unique occasion to perform a global reflection on thermonuclear fusion which is summarized in this paper. We first consider the case for fusion energy then move on to the scientific achievements during the past five decades. Finally, the lessons drawn from the past give a framework to consider the challenges ahead of us. The 1958 pioneers had the vision of the vital importance of international collaboration to succeed in this unique endeavour. Fifty years later, this vision has amply proven its worth. Looking at the way forward, this vision constitutes a strong basis to harness fusion energy in the decades to come.
\end{abstract}

PACS numbers: 28.52.- s, 52.00.00, 52.55.-s, 52.57.-z, 89.30.Jj

\section{Energy: a major challenge for this century}

Energy has now become a major challenge for this century which needs to be addressed as a matter of urgency. At present, the world relies primarily on fossil fuels: petrol, gas and coal. They provide primary energy production at a rate of about $86 \%$. Nearly $70 \%$ of the fossil fuels originate from hydrocarbons in the form of gas and petrol. We have entered into a new phase where consumption of the hydrocarbons exceeds new discoveries and the margin between production capacity and demand is becoming very small. Consequently the age of cheap oil is over and producers are increasingly considering protecting their reserves. The price of oil has become very volatile as a direct consequence of the much reduced production margin.

Between 2000 and 2006 the world's primary energy consumption has increased at a rate of about $2 \%$ per year. Strikingly, the increment comes, to a large extent, from burning coal. In 2006 for instance, China increased its electrical capacity by $105 \mathrm{GW}, 90 \%$ of which is produced by coal power plants. This is nearly identical to the total installed electrical capacity of France. Burning coal releases the highest amount of carbon dioxide and other greenhouse gases. As plans for carbon sequestration on an industrial scale are expensive and remote, the consequences for the environment and for global warming of this return to coal are extremely worrying.

It is essential and urgent for the benefit of our descendants to do better than a return to coal. No simple solution will resolve this challenge. Clearly a vast and coherent plan comprising energy wastage avoidance, the development of renewable energies, carbon sequestration and nuclear energies should be vigorously pursued.

\section{The case for fusion}

A deployment of various forms of nuclear power therefore appears necessary in particular for base load electricity production. Fission has amply shown its worth in countries which put a strong emphasis on safety and security and adhere strictly to the non-proliferation treaty. It promises a bright future when these conditions can be guaranteed. Fusion has still a long R\&D way to go to be available industrially but would offer three major advantages: the absence of very long lived radioactive waste (figure 1) would considerably ease the waste management aspects, the primary fusion fuels (deuterium and lithium) are essentially inexhaustible and a Chernobyl-like runaway situation is physically impossible as there is no more than a minute of burn of $\mathrm{D} / \mathrm{T}$ fuel in a fusion reactor which needs to be continuously refilled with a mixture of $\mathrm{D} / \mathrm{T}$ gas. The reaction would therefore be automatically quenched by air ingress in the case of a serious accident.

\section{The discovery of fusion}

The first man-produced fusion reactions were performed in 1932 [1] by Rutherford and his team in Cambridge, UK. In this famous experiment, not only did they observe the fusion of deuterium atoms but they also discovered two new fusion born nuclei, ${ }^{3} \mathrm{He}$ and tritium. It was a time when you could and should demonstrate your findings publically (see [2] and figure 2). Incidentally, the photograph shows that safety aspects were not an overwhelming consideration in these times! During his demonstration, Rutherford did mention that each fusion reaction produced a large amount of energy, but there was overall a net loss of energy because of the low fusion 


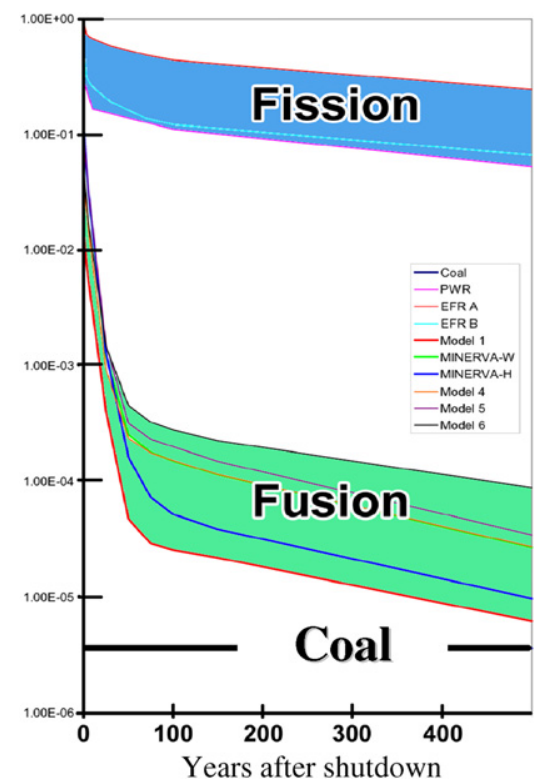

Figure 1. Relative radio toxicity of fission and fusion reactors versus time after shutdown. The bands correspond to differences in the fuel cycle (reprocessing) for fission and to the choice of structural material for fusion. The bottom black line is the radio toxicity of coal.

probability of the deuterium atoms he had accelerated against the deuterium target. In his talk he dismissed nuclear energy as 'moonshine'!

Nuclear physics was brilliantly launched by the Cambridge team and went on making progress with huge strides. In 1934, Bethe and Peierls developed a theory of the nucleus of the deuteron [3]. By 1938, Hans Bethe (figure 3) had predicted the cross-section of many nuclear interactions. This allowed him to establish that the stars were powered by fusion processes. In the enormous ball of gas which constitutes a star, initially mainly hydrogen, a complex fusion cascade is taking place transforming, over billions of years, first hydrogen into helium then into progressively heavier nuclei.

By the early 1950s, the basic properties of fusion of light atoms were sufficiently well known for John Lawson (figure 3) to formulate his famous necessary criterion [4] to produce net energy from a fusion reactor based on confinement$n \tau_{\mathrm{E}} \geqslant 1.5 \times 10^{20} \mathrm{~m}^{-3} \mathrm{~s}$-where $n$ is the ion density and $\tau_{\mathrm{E}}$ is the energy confinement time of the fuel ions. He also stated that the temperature needed to be about $10 \mathrm{keV}$ (100 millions degrees). Typically, the conditions to be reached are $n \sim 10^{20} \mathrm{~m}^{-3}, \tau_{\mathrm{E}}>1.5 \mathrm{~s}, T>10 \mathrm{keV}$. Achieving the density was relatively easy. The temperature seemed to be a daunting task but several methods have now successfully reached and even exceeded this value. However, the real challenge was to be the energy confinement time. It is indeed the main objective of ITER to exceed for the first time in a magnetic confinement device the value required for net energy production.

\section{Three ways for fusion}

The stars are natural thermonuclear reactors which benefit twice from gravitation. Initially, gravitation collapses a huge hydrogen cloud, providing during this process the heating required for reaching ignition conditions. Then, during the burn, it balances the expansion force resulting from the huge internal pressure $\left(\sim 10^{9}\right.$ atm. $)$. For a sun-like star, equilibrium is achieved for a diameter of about one million kilometres.

On earth, a force stronger than gravitation is needed so that the burn can occur on a much smaller distance. This can be done transiently by fast compression of a tiny D/T fuel cell using, for instance, laser beams. The pressure needs to be well over the billion bar mark so that a significant fraction of the fuel can burn during the compressed phase when inertia maintains a sufficient pressure. In reactors, it will be necessary to repeat this process at a rate of about $10 \mathrm{~Hz}$.

The other way to achieve controlled fusion on earth is by using a magnetic field to create an immaterial confinement bottle. In this case, the plasma can be confined in the steady state. The fuel pressure is limited to a few bars both by the values of the magnetic fields which can be achieved in practice, and by the thermal loading of the plasma facing components. Several magnetic topologies have been tested during the early days of fusion research and toroidal confinement devices are now clearly the preferred choice. The best performances have been obtained so far in the tokamak configuration which has naturally been chosen for the ITER project (figure 4).

\section{1958: fusion research is declassified}

The USA first demonstrated the military potential of fusion energy with the H-bomb test on 1 November 1952. The blast was hundreds of times more powerful than the A-bomb explosion at Hiroshima. A Russian demonstration followed less than a year later. Scientists were, at this time, reasonably confident that fusion energy could also be harnessed for peaceful purposes, following the path indicated by Lawson. However, the first tests of fusion machines in 1954-1958 such as the Perhapsatron $[5,6]$ in the US or Zeta $[5,6]$ in the UK clearly showed that plasmas did not let themselves be easily confined by magnetic fields. These early attempts were marred by major plasma instabilities which were poorly understood at the time.

In 1955, the conference 'Atoms for Peace' took place in Geneva. It created great hope for the potential benefits of peaceful nuclear cooperation. With regard to fusion, the chairman of the conference, Homi Bhabha, said 'I venture to predict that a method will be found for liberating fusion energy in a controlled manner within the next two decades.' During a famous visit at Harwell in 1956, I.V. Kurtchatov lifted a part of the veil of secret on the Soviet fusion enterprise. It suggested a willingness to open up the USSR research on fusion. Indeed, controlled thermonuclear fusion was to become a significant item for the second 'Atoms for Peace' conference to be held again in Geneva in 1958.

One hundred and eleven papers on fusion energy were presented at this first IAEA conference held just 50 years ago. Scientists from countries belonging to the IAEA organization met in an enthusiastic atmosphere of openness (figure 5). At the start of the proceedings, fusion was formally declared declassified by the USA and the UK following a similar announcement made a little earlier by the USSR.

The conference proceedings remind us that the scientific leaders of the main delegations were much less optimistic 


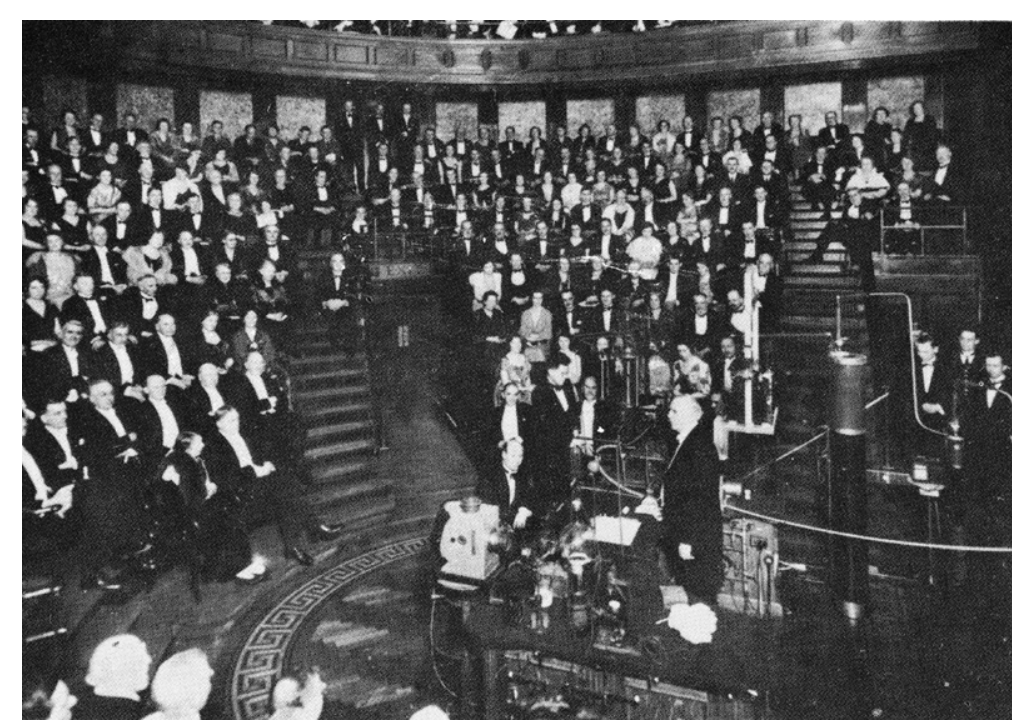

Figure 2. Rutherford demonstrating fusion of deuterium atoms at the Royal Institution in 1934.

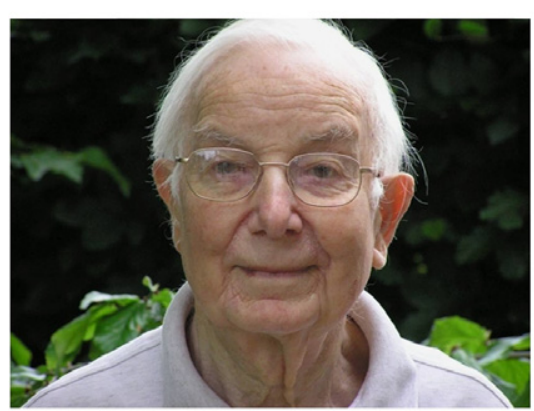

J. D. Lawson 1923 - 2008

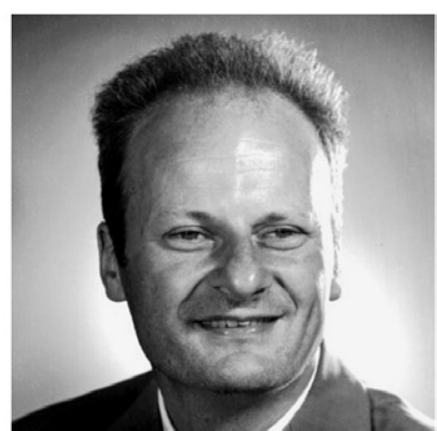

Hans Bethe 1906 -2005

Figure 3. Two fusion pioneers.

\section{Three ways for fusion}

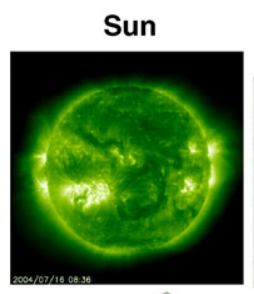

1

\begin{tabular}{l|c|}
\hline Confinement: & gravitation : \\
\hline Dimension: & $1.310^{8} \mathrm{~m}$ \\
\hline Duration: & $310^{16} \mathrm{~s}$ \\
\hline Pressure: & $10^{9} \mathrm{~atm}$ \\
\hline
\end{tabular}

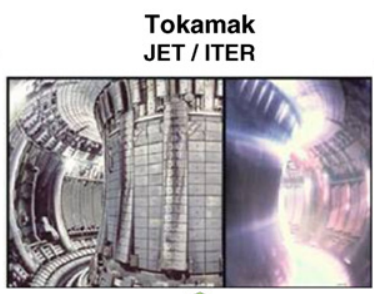

1

magnetic :

$10 \mathrm{~m}$

$400 \mathrm{~s}$

$2 \mathrm{~atm}$

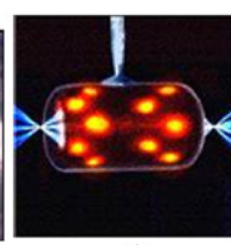

U

inertial :

$10^{-2} \mathrm{~m}$

$10^{-8} \mathrm{~s}$

$10^{11} \mathrm{~atm}$

Figure 4. Three ways to fusion power.

than Bhabha 3 years earlier. Edward Teller (figure 6) from the United States said that the state of controlled fusion was 'similar to the stage at which flying was about 100 years ago' and observed that the technical difficulties of fusion 'are likely to make the released energy so costly that an economic exploitation of controlled thermonuclear reactions may not turn out to be possible before the end of the twentieth century'. Similarly, when reviewing work in the Soviet Union,
Lev Artsimovich (figure 6) stressed that the difficulties lying ahead are so large that 'worldwide collaboration is needed for progress'.

This statement from Artsimovich had a tremendous impact and still resonates in our ears. Soon after, a network of collaborations under the auspices of the International Atomic Energy Agency (IAEA), the International Energy Agency (IEA) and EURATOM (the European atomic-energy 

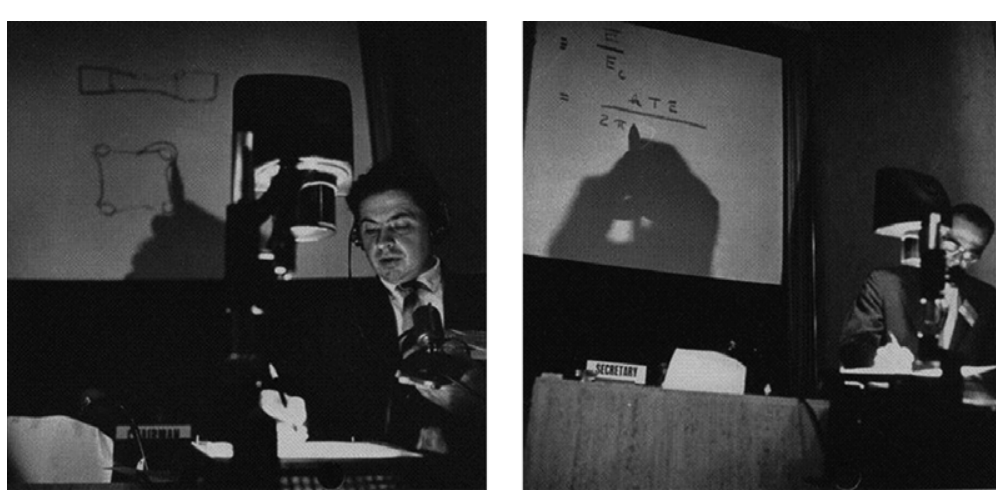

Figure 5. Two presentations at the first FEC conference 50 years ago: left: B. Kadomsev lecturing on plasma instabilities. Right: L. Spitzer explaining the stellarator concept.
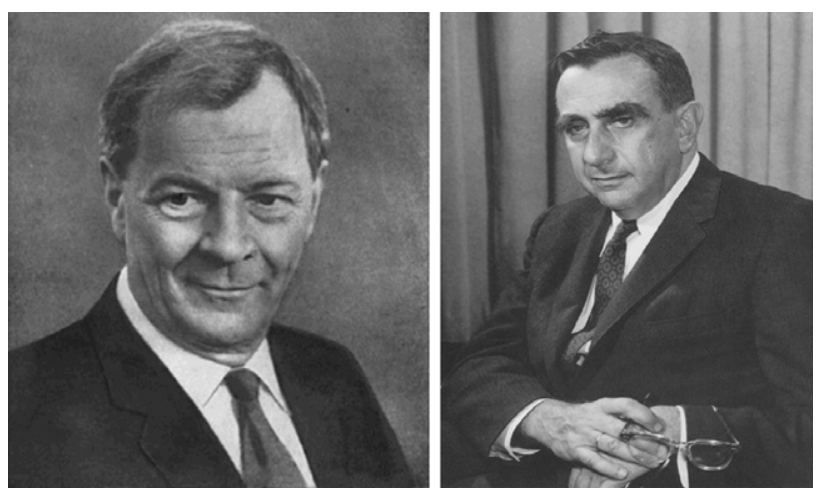

Figure 6. Lev Artsimovich and Edward Teller.

community) was established in the domain of magnetic fusion. Today these structures are very active in coordinating fusion research and the spirit of 1958 is still very much alive.

It is also after this memorable first fusion energy conference that several new important fusion laboratories were created, notably in France, Germany, Japan and in the USA. Attention was given to establishing strong links between these labs and the universities and other multidisciplinary research organizations. Good examples of this are the John Jay Hopkins laboratory, San Diego, inaugurated in 1959 by Creutz, Bohr and Kerst and DRFC (Département de Recherches sur la Fusion Contrôlée) in France, which became in 1959 the first national laboratory under contract of association between CEA and EURATOM. Similar contracts of association were progressively concluded with all laboratories coordinating research in fusion science and technology. Today, 26 contracts of associations are operating in 24 countries of the European Union and in Switzerland.

\section{Scientific milestones in magnetic fusion}

Table 1 is a brave attempt to summarize the scientific milestones during the five decades since the 1958 conference. A single table is obviously unable to mention all the good science done during the half century. It does, however, give the general trends.

During the first decade 1958-1968, the foundations of a new science, plasma physics, were laid. Plasmas soon appeared to be a complex media dominated by collective interactions between particles, waves and fields. Its basic mechanisms were established theoretically. A number of possible confinement structures were identified: mirror machines and toroidal magnetic confinement devices appeared to be promising. However, new instabilities were discovered at a depressing rate! Global MHD instabilities were the worst: when plasmas experienced a concave line averaged magnetic field curvature, the instability could push it across the field lines very rapidly at the speed of sound! Early simple mirror machines had such bad curvature and the energy confinement time was orders of magnitude lower than what was expected on the basis of particle collisions. A significant success was recorded when the Kurtchatov Institute showed that a quadrupolar field, the 'Ioffe bars', added to the simple mirror field could change the curvature from bad to good. The large scale instabilities disappeared and confinement improved. However, the more stable plasma revealed a new weaker type of instability, the 'microinstabilities' fed by free energy in velocity space, which could precipitate particles in the loss cone of the configuration. Even today, it has not been possible to eliminate this loss in a satisfactory way for a reactor.

Toroidal devices were also experiencing the disappointment of low confinement results. Initially, the use of a glass or even quartz vessel would lead to plasma contamination. This was resolved by using cleaned metallic walls and improved pumping. Temperatures did increase and neutrons appeared. The ZETA machine in Harwell, a toroidal pinch, even claimed obtaining neutrons of thermonuclear origin. Alas, this proved to be an error of interpretation. Neutrons came from interaction of accelerated ions with internal components. The effect was also the result of violent instabilities. In Princeton, on the other side of the Atlantic, the ingenious stellarator concept invented by Lyman Spitzer was actively explored. The vertical drift due to toroidal magnetic curvature was cancelled by twisting the magnetic lines of force either with a 'figure 7' configuration or using helicoidal windings around a more conventional vessel shape. Results were also disappointing but the reason only became clear a few years later: error fields would destroy the magnetic surfaces due to resonant effects.

The breakthrough on toroidal devices came again from the Kurtchatov Institute. At the 1968 FEC in Novosibirsk, Lev Artsimovich impressed everyone present when he reported that tokamak $\mathrm{T} 3 \mathrm{had}$ reached a temperature of $1 \mathrm{keV}$ and a confinement time of some milliseconds which far exceeded all values previously obtained. The demonstration came with 
Table 1. A few key milestones during five decades of research on magnetic confinement.

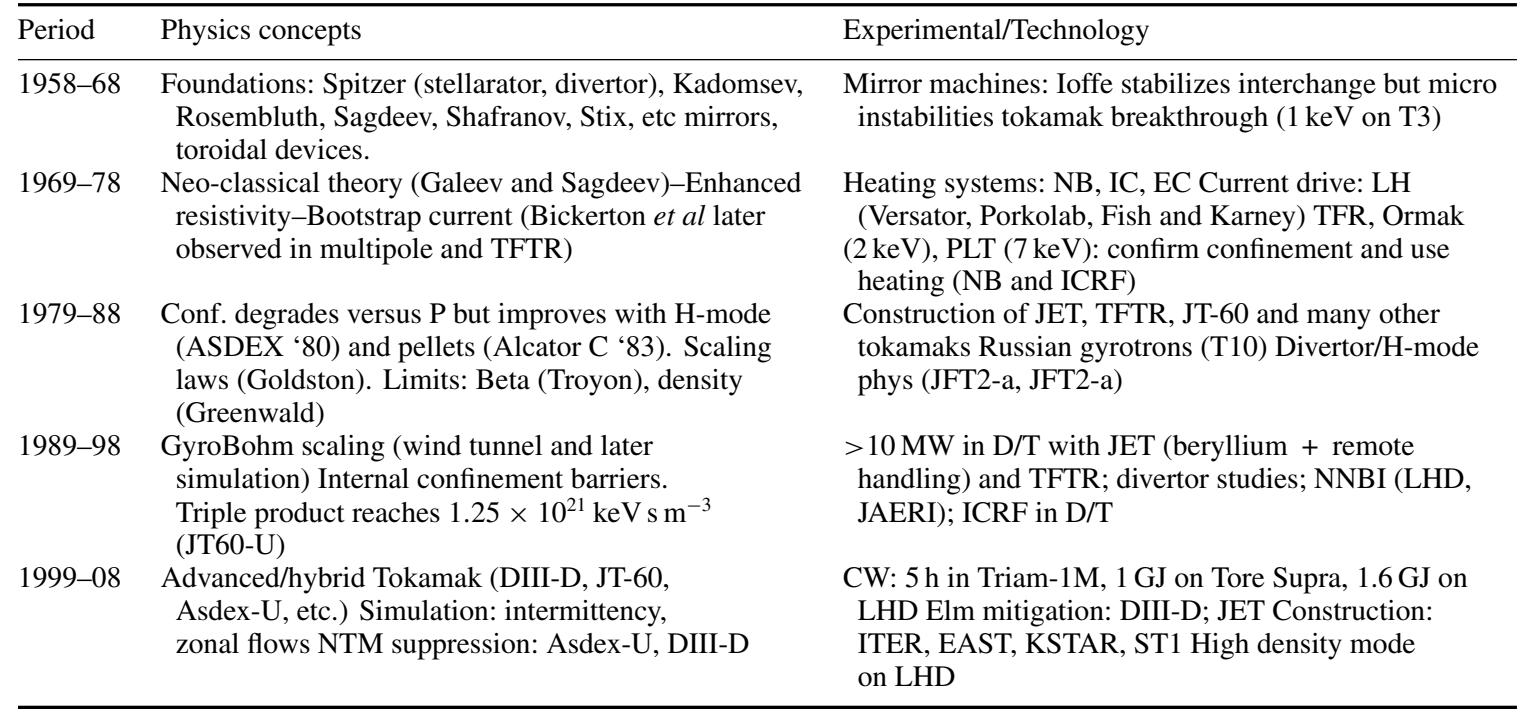

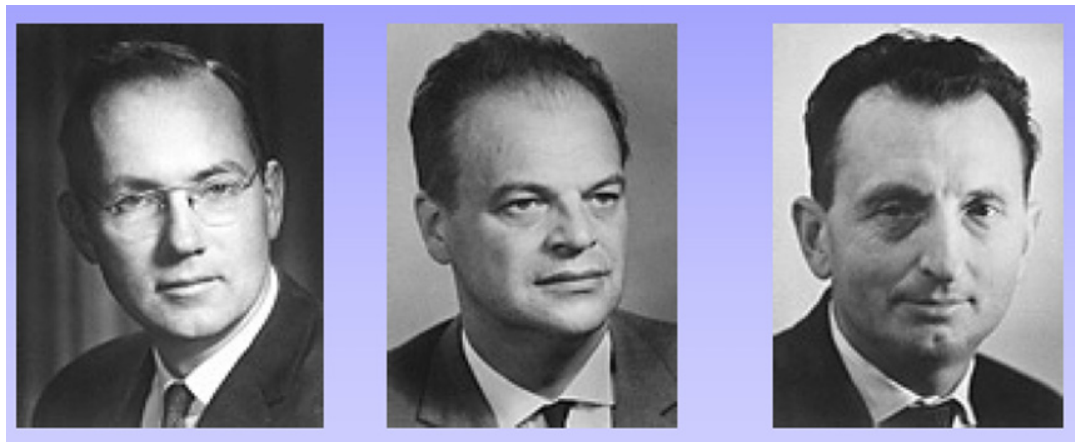

Figure 7. C. Townes, N. Basov and A. Prokhorov, the LASER pioneers.

the explanation that limiting the plasma current so that the edge $q$ value (inverse rotational transform) was greater than 3 would eliminate the most damaging MHD instability. All doubts on the validity of the results were removed when a British team responded to the invitation for measuring the temperature using the newly developed Thomson scattering technique. They brilliantly confirmed the claim from their Russian colleagues. The western laboratories were convinced and tokamaks would soon bloom everywhere with the help of lectures given by Artsimovich himself. In Saclay in 1968, the author of these lines and several of his colleagues benefitted greatly from such high level training. From this date, tokamaks became dominant. In France in particular, the entire research group in Fontenay aux Roses rapidly concentrated its effort on this configuration.

During the decade 1969-1978, tokamak devices were built in all industrial countries. They confirmed the confinement potential of the configuration. TFR [7] in Fontenay aux Roses was the first in the western world to go beyond the Kurtchatov results thanks to its larger plasma current. Confinement did increase with size and field strength as hoped. However, the temperature could not go much above $1 \mathrm{keV}$ as the ohmic heating power diminished as $T_{\mathrm{e}}^{-3 / 2}$ and the ion temperature lagged behind. The need to add powerful external sources of plasma heating became the next major challenge to overcome. Intense beam sources were first developed and injected in the tokamak after neutralization in a gas cell. Ormak in Oak Ridge and TFR first reached an ion temperature of $2 \mathrm{keV}$. These results were exceeded a few years later by the even larger PLT tokamak which reached $7 \mathrm{keV}$. In parallel, the science of RF heating, initially developed by T. Stix, made considerable progress. Highly localized heating could be achieved either with the ion cyclotron range of frequency (ICRF) operating in the $50 \mathrm{MHz}$ domain or with electron cyclotron heating (ECRH, 50-100 GHz). Pioneering demonstrations of RF current drive were also realized with lower hybrid waves $(\mathrm{LHCD}, \sim 1-$ $4 \mathrm{GHz}$ ). By the end of the decade, several methods could be used for heating plasmas towards thermonuclear temperatures via either the electron or the ion channels.

On the theoretical front, the 'neoclassical theory' [8] represented major progress in understanding transport driven by collisions in full toroidal geometry. This led, inter alia, to the prediction of the 'bootstrap current' [9] which was later observed in a toroidal multipole (Zarnstorff et $a l$ ). However, the rate of transport across the field lines of electron heat predicted by the theory was always exceeded in experiments by several orders of magnitude. But the phenomena related to the movement of particles along the field lines such as the increased central resistivity due to trapped electrons or the bootstrap current mentioned above were confirmed by experiments. It emerged that the plasma turbulence would affect only the much 
slower diffusion across the field lines and preferably via the electron channel.

The first part of the next decade, 1979-88, focused on the construction of JET, TFTR and JT60. They relied on a bold increase in size to improve confinement. TFTR put more emphasis on having a large beam power to take advantage of non-thermal beam-plasma fusion reactions while JET, conceived by Rebut and his team, put its emphasis on plasma volume and on a D shape cross section to increase the confinement time and the true thermonuclear yield. The mission of JET [10] included observing self heating by fusion born $\alpha$ particles. This implied operation in tritium and all related nuclear aspects including remote handling of in-vessel components. During the same period, the requirement for a divertor to control the exhaust of heat and particles became apparent and several machines concentrated on this aspect, notably JT60 in Japan and ASDEX and Dite in Europe. The construction of these machines was well under way when the news came [7] of the degradation of confinement with additional heating power. First observed with NBI heating, the phenomenon was soon reproduced with RF heating. A first international database gave an empirical scaling [11] of confinement degradation which was later referred to as the 'L-mode'. It was depressing news as this scaling predicted that a tokamak would have to be embarrassingly large to produce a net energy gain. Only a short time later, however, ASDEX discovered the H-mode [12], a confinement barrier spontaneously occurring at the plasma edge above a certain power threshold. Again this phenomenon proved to be universal. It was soon reproduced and documented in other tokamaks. In particular, DIII-D identified the change in $E \times B$ shear at the $\mathrm{L}$ to $\mathrm{H}$ transition. The conditions for its occurrence and the scaling of both the 'H-mode' confinement and of its power threshold were established during the following two decades. H-mode scaling is today a well confirmed basis for the ITER design.

Other essential contours of the tokamak operating space were defined from a combination of theoretical and experimental studies. The limit in normalized plasma pressure 'Tryon's limit' [13] and density 'Greenwald limit' [14] are the most important ones. They can only be exceeded when certain precise conditions are met.

The following decade, 1989-98, contains the historical milestone of the first experiments using a deuterium/tritium mixture. Pure deuterium runs predicted that fusion power in the megawatt range would be generated when a balanced $\mathrm{D} / \mathrm{T}$ mixture was used. To perform such an experiment requires considerable technological development; firstly on tritium containment and safety, on remote handling of the machine core, on adapting heating systems to tritium and finally on diagnostics which have to withstand a large flux of $14 \mathrm{MeV}$ neutrons. The resounding success of these experiments (figure 8) played a major role in making the case for the ITER project.

This fourth decade was also marked by the creation of an international confinement database under the auspices of IAEA which led to non-dimensional scaling laws based on first principles and on a 'wind tunnel' approach. The 'Gyro Bohm' scaling character of H-mode confinement was confirmed. The laws indicated the dimensions of ITER required for reaching

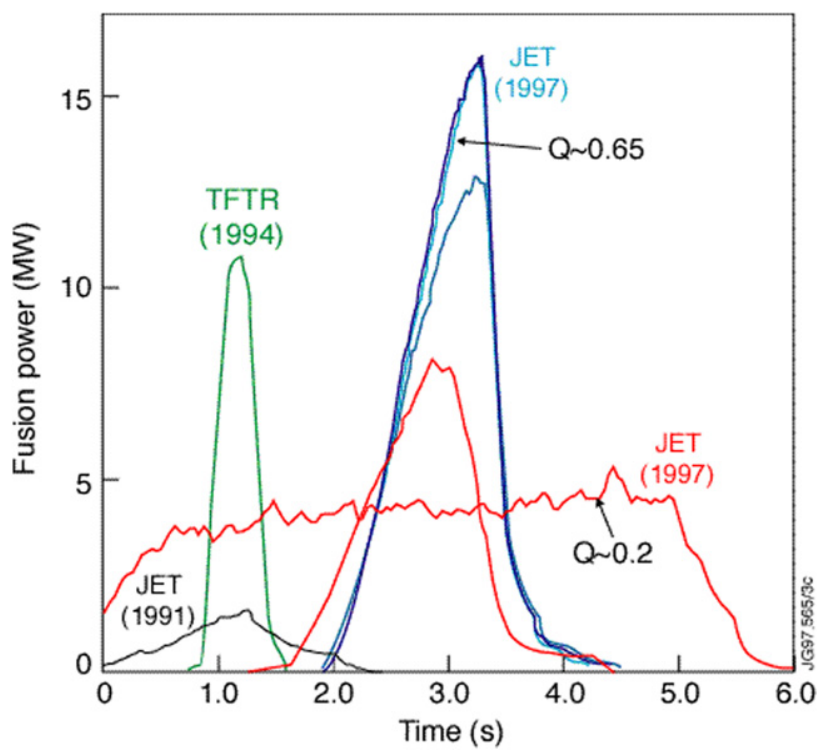

Figure 8. Evolution of fusion power produced in JET and TFTR using D/T fuel.

its $Q=10$ objective. The discovery of internal confinement barriers generated either with pellets (JET) or by current profile control (DIII-D, JT60-U, etc) is also a highlight of this decade and is expected to have important consequences in the future. Also outstanding is the progress achieved in divertor concepts and operation and in the field of additional heating (ICRF in $\mathrm{D} / \mathrm{T}$ plasmas, negative ion based neutral beam heating and ECRH gyrotron sources).

The last decade, 1998-2008, has been dominated by the launch of the ITER programme. But this is certainly not the only item of considerable importance. A broader approach to magnetic fusion between Europe and Japan is closely associated with this launch and includes major steps towards the development of fusion materials (IFMIF), computing with the new International Fusion Energy Research Centre (IFERC) and steady state tokamak operation with the construction of a new Japanese large superconducting device, JT60-SA. The decade is also remarkable for the construction, notably in Asian countries, of new facilities using superconducting magnets with the ultimate aim of steady state operation. These new devices have either started operation, for example EAST in China and KSTAR in Korea, or will be commissioned in the coming years (ST1 in India and W7X in Germany).

The physics milestones which have been reached during this period are equally outstanding. They concern a large range of experimental physics aspects including new modes of operation (hybrid mode, ASDEX-Upgrade, DIII-D) with the potential of steady state using reduced external current drive power, stabilization of NTM instabilities occurring at high pressure (ASDEX-Upgrade, DIII-D), ELM mitigation using ergodic external perturbations of the edge magnetic field (DIII-D, JET) and high density mode of operation in the Large Helical Device (LHD). Modelling and theory have given us new understanding on how particle and energy transport proceeds with the formation of large scale eddies moving across the field lines in an intermittent manner. Computer simulations of realistic cases have made huge 
progress although their predictive capabilities for large devices such as ITER and the reactor are still insufficient.

Finally, the decade will be remembered for making considerable advances in long pulse operation with tokamak and stellarators-TRIAM-1M in Japan could operate for up to $5 \mathrm{~h}$ in full steady state operation using LHCD-Tore-Supra exceeded first the mark of $1 \mathrm{GJ}$ of energy during 6 min pulses with about $3 \mathrm{MW}$ of LHCD and more recently the Japanese LHD increased this landmark to $1.6 \mathrm{GJ}$ using ICRH. These results imply an impressive progress in a broad range of technological fields (materials, actively cooled components, real time control, etc). It should be stressed, however, that there is still a long way ahead of us towards the $400 \mathrm{GJ}$ specified for ITER with the additional burden of the nuclear environment.

\section{Scientific milestones in inertial fusion using lasers}

The fundamental research of C. Townes, A. Kastler, N. Basov, A. Prokhorov and T. Maiman in the field of experimental physics, which led to the discovery of the maser and the laser in the 1950s, have opened vast new horizons in physics. One of these new horizons was the possibility to shine huge power densities on matter to reach the conditions for thermonuclear fusion. In the 1960s, the possibility of compressing micro targets by a programmable laser driver began to be explored. In 1970, the Limeil team in France reported in Physical Review $A$ the first production of DD fusion neutrons in a $1 \mathrm{keV}$ plasma heated by a powerful laser. A major step in the ICF programme took place in 1972, when John Nuckolls of the Lawrence Livermore National Laboratory (LLNL) published a seminal paper in Nature [15] that predicted that ignition could be achieved with laser energies about $1 \mathrm{~kJ}$, while 'high gain' would require energies around $1 \mathrm{MJ}$. High energy ICF experiments with multi-hundred joules per shot began in the early 1970s, when lasers of the required energy and power were first designed. One of the earliest large scale attempts at an ICF driver design was the Shiva laser, a 20-beam neodymium doped glass laser system built at LLNL that started operation in 1978. Shiva was a design intended to demonstrate compression of fusion fuel capsules to many times the liquid density of hydrogen. Indeed Shiva succeeded and compressed its pellets to 100 times the liquid density of deuterium. However, due to the laser's strong coupling with hot electrons, premature heating of the dense plasma (ions) was problematic and fusion yields were low. This pointed to the use of optical frequency multipliers as a solution which would triple the infrared light frequency from the laser into the ultraviolet at $351 \mathrm{~nm}$. Newly discovered schemes to efficiently frequency triple high intensity laser light discovered in 1980 enabled this method of target irradiation to be experimented with the Nova laser designed with 10 times the energy of Shiva. This was the first design with the specific goal of reaching ignition conditions.

Nova failed in its goal of achieving ignition due to variation in laser intensity in its beams which resulted in large non-uniformity in irradiation smoothness at the target and asymmetric implosion. Between 1980 and 1995, however, the experiments Nova and Omega in the USA, GXII in Japan and Phébus and Luli in France led to a much greater understanding of the process of implosion. The way forward clearly appeared to increase the uniformity of irradiation through beam smoothing techniques to reduce the effects of the Rayleigh-Taylor instability. It was also concluded that the laser energy on target needed to be increased further towards the MJ range.

The resulting concepts have been the design basis for two new very large facilities presently under construction: the National Ignition Facility (NIF) in Livermore, USA and the Laser Mega Joule (LMJ) in CESTA, France. NIF started construction at LLNL in 1997. Its main objective will be to operate as the flagship facility of the 'nuclear stewardship programme'. It is scheduled for fusion experiments to start in 2009 when the remaining lasers in the 192-beam array are installed. The first attempts at ignition are scheduled for 2010. Similarly, LMJ is funded from the defence budget. Construction of its 240 beams, grouped in 60 quads focused by gratings, started in 2003 and should be completed in 2012. The technological choices of the LMJ have been recently validated by the successful operation since 2004 of the LIL laser, an 8 beam prototype of LMJ which has exceeded its nominal parameters (60 kJ in UV).

A more recent development is the concept of 'fast ignition' [16] which may offer a way to directly heat the high density fuel after compression, thus decoupling the heating and compression phases of the implosion. In this approach, the target is first compressed using a driver laser system, and then when the implosion reaches maximum density, a second ultrashort pulse ultra-high power (petawatt) laser delivers a single pulse focused on one side of the target, heating it to start fusion ignition. Several projects are currently underway to explore the fast ignition approach, including upgrades to the OMEGA laser at the University of Rochester and the GEKKO XII device at the University of Osaka in Japan.

New facilities based on this concept are now being projected. They are known as the High Power laser Energy Research facility (HiPER), an EU study, and FIREX II in Japan. If successful, the fast ignition approach could lower by about an order of magnitude the total amount of energy needed to be delivered to the target; whereas NIF uses UV beams of $2 \mathrm{MJ}$, HiPER plans for a driver of $200 \mathrm{~kJ}$ and a heater of $70 \mathrm{~kJ}$, yet the predicted fusion gains are expected to be higher than NIF.

\section{International collaboration}

Ever since Artsimovich's statement at the first FEC, international collaboration has been a trademark for fusion research. It has been shaped under the auspices of 3 organizations.

Firstly, the IAEA has set up an International Fusion Research Council (IFRC) which oversees a number of joined activities organized by the agency: the biannual Fusion Energy Conference attracting well over 500 participants, a number of technical meetings focussed on topical issues, joined projects and training, maintenance and updating of the atomic and molecular data for fusion and, this is in everybody's mind, the IAEA has provided the auspices for ITER through all its phases (conceptual design, engineering design activities, negotiations and presently the custody of the ITER documents).

Secondly, and as a direct consequence of the 1958 conference, EURATOM has constructed an efficient network of European laboratories linked by contract of association. 
The contract scheme provides a financial contribution from the European Community in exchange for jointly setting up priorities and sharing research results as well as providing mobility of staff. Started in 1958 with the EURATOMCEA association, the network has now 26 contracts linking essentially all laboratories from 24 countries involved in fusion (including Switzerland). It now fully includes the arrival of the new European states. The strength of this European fusion community is well illustrated by the success of the joint construction and exploitation of JET and by its concerted effort to offer a suitable site for ITER construction.

Thirdly since 1975, the IEA coordinates via the Fusion Power Coordinating Committee (FPCC) implementing agreements between laboratories of member states on specific subjects. The main areas of collaboration have been plasma physics, materials, safety, environmental and socio-economic aspects. The agreements have been successful for providing and organizing staff exchanges, joint experiments and, in a few cases, hardware exchange. The conceptual studies for IFMIF (International Fusion Material Irradiation facility) are a good illustration of a programme which could not have succeeded to the same extent without this framework. With the arrival of ITER and the considerable evolution of the world's fusion programme, it is clear that the organization of the implementing agreements needs now to reflect these profound changes.

In addition and in a transverse capacity to these three large organizations, the 'International Tokamak Physics Activities', (ITPA), coordinates and stimulates voluntary joined activities in support of ITER. Created in 1992 as 'ITER Expert groups', they became ITPAs in 2001, reporting to IFRC and FPCC, and are now under the chairmanship of the ITER organization. The ITPA members come from the 7 ITER partners. They are organized in topical groups focusing on assembling databases and providing projections for ITER performance and operating domains. It is certainly not an exaggeration to stress their vital role in providing fundamental scaling laws, validating computer codes and requesting joint experiments on crucial ITER issues from existing machines via the IEA implementing agreements mentioned previously. Figure 9, which shows the confinement scaling law derived from the international database, illustrates well one of the many successes of this activity. In 2008, the ITPA published in the Nuclear Fusion journal 'Progress in the ITER Physics Basis', a very large paper [17] gathering the best information worldwide on the subject. The success of this publication was immediately impressive: 20000 downloaded chapters had been registered only a few months after being made available in electronic form.

\section{Lessons from the past and the way forward}

\subsection{Physics}

The past five decades have been dominated by the challenges of plasma physics, but this situation has now changed as a strong scientific basis for fusion is now available. The linear phase of complex phenomena such as stability and heating processes are well described by physics solutions based on first principles. Validated codes benchmarked worldwide are now available for optimization purposes. However, heat and

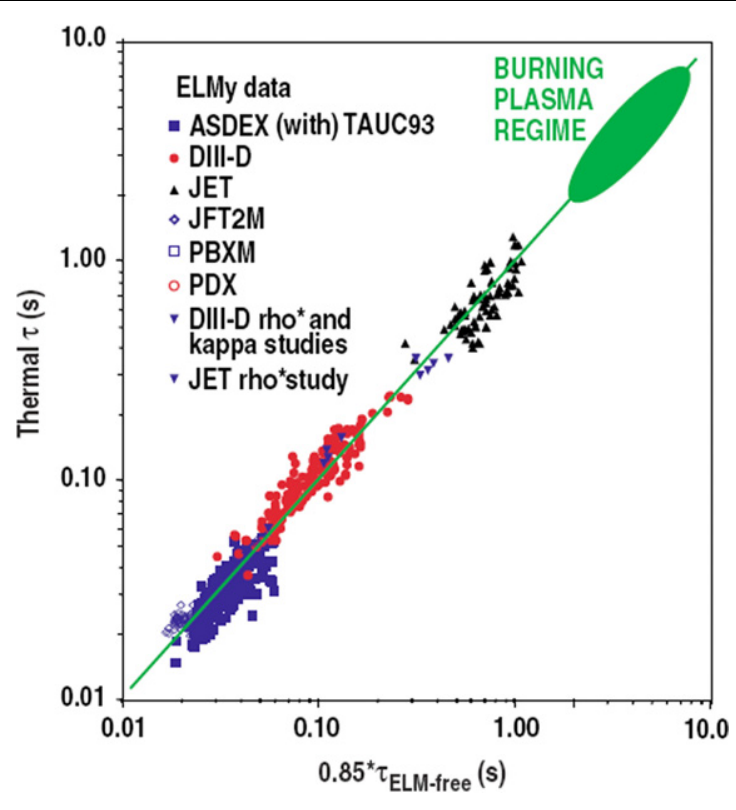

Figure 9. Tokamak confinement scaling law derived from the database constructed by the ITPA.

particle transport generally proceeds through fully developed non-linear turbulence driving multiscale vortices and inducing zonal flows. These fundamental processes have only been recently observed both in computer simulations and in tokamak experiments. It is fair to say that the predictive capability of this fascinating science is presently insufficient and that considerably more effort is required to acquire such capability for a precise scaling to the fusion reactor. Surprises, good or bad, from new physics occurring at reactor parameters cannot be excluded. In the meantime, global scaling laws have been provided by the non-dimensional 'wind tunnel' approach based on the international database as illustrated in figure 9. This method has amply proven its worth in similar fluid or hydrodynamics problems, notably in the design of aircraft or boats. Much more physics insight and scaling knowledge is expected from the work of the ITPAs, in particular in the area of advanced tokamak operation and their strengthening is certainly required. In combination with simulations based on first principles, the enlarged international databases should bring improved predictability and the required insight for optimization towards the reactor.

A new physics challenge of toroidal devices is expected to be the stability of burning plasmas during long pulse operation. For the first time in ITER, self heating by fusion born $\alpha$ particles will be dominant. The stability of the $\alpha$ particle population will have consequences on the current profile which itself will modify transport which in turn will close the feedback loop by changing the fusion rate and the $\alpha$ population. Although not necessarily unstable, the behaviour of this loop over very long pulses is a main issue. It needs to be addressed as a matter of priority by developing the adequate modelling tools and by providing the adequate diagnostics for ITER.

\subsection{Technology}

In fusion devices, engineering and physics are highly integrated. To a large extent the machine itself as a whole 
is the experiment. This is particularly true in tokamaks. As an example, the causes and consequences of disruptions bind closely together the technology of the vacuum vessel and the physics of the halo currents flowing between the plasma and the vessel.

For both magnetic and inertial fusion, safety and materials are key issues. Helium formation in structural materials under intense $14 \mathrm{MeV}$ neutrons irradiation will require the development of adequate solutions. This will require both modelling and considerable effort to validate choices using a dedicated irradiation facility.

For ICF reactors, progress by many orders of magnitude must be accomplished in the area of highly repetitive lasers with good plug to target efficiency and on low cost targets. To obtain ignition from a single pulse will be a great achievement but to produce a second one just $100 \mathrm{~ms}$ later with an overall energy gain is a much tougher challenge.

On the MCF side, success will depend greatly on the capability to run either in true steady state or in a repetitive long pulse mode with a high duty factor. The challenge here is to first demonstrate the capability to account for the precise distribution of all the power generated by the device and to cool all parts of the machine adequately despite peak power loads which can, in places, exceed $10 \mathrm{MW} \mathrm{m}^{-2}$. The second part of the $\mathrm{CW}$ operation challenge is to minimize the power consumed by auxiliaries (cooling pumps, cryo-magnetic systems, current drive if any, heating and control systems). There is a long way from the present situation in fusion to the state of fission power plants where the internal power recirculation for feeding auxiliaries is only on the order of $3 \%$ to $4 \%$. Therefore the operation in $\mathrm{CW}$ or high duty repetitive cycle is considered as a major future era for fusion engineering.

\subsection{Project organization}

Project organization will play a major role in the success of the construction of ITER and the accompanying devices. Here again let us draw on the lessons from the past. In 1986, the Director of JET, Hans Otto Wüster, was invited by the US Congress to testify on the success of the JET construction. In his own words 'JET could succeed because it was given the power to manage'. The JET Council sets 'what' and the JET Director, and only he, proposes 'how' and manages the entire construction budget. The council approves, or not, the Director's proposal.

The ITER organization is different: ITER IO only manages directly a small part of the budget and the 7 domestic agencies procure 'in-kind' most of the machine. This additional layer does increase the complexity of the interfaces which remain the full responsibility of IO. Many are doubtful that such a system can work. However, successful precedents of a similar organization do exist. In CERN, in particular, large experimental set-ups such as Atlas, have been constructed in this way. Indeed, the analysis of the construction of the large superconducting coils of Atlas [18] shows that the construction could be completed on time with a modest cost increase of $16 \%$. But this success requires that the central team be strong to cross-check procurements at all time and levels. The devil is in the 'classical' details and not in the high technology part; most of the problems were encountered in welds. The people involved in this 'in-kind procurement' should recognize that this complex organization which forces people to pull together knowledge as well as material resources can have highly beneficial consequences.

\section{The coming decade}

For both MCF with ITER and ICF with NIF and LMJ, the coming decades will be dominated by the demonstration of burning plasmas with these three very large facilities. We note that what the ICF community calls 'ignition' is in terms of power gain, similar to the $Q=10$ objective of ITER. The success in reaching this common objective will be equally crucial for both schemes. On the physics side, the new frontiers of the burning plasma state will be explored for the first time. The experimentalists will venture into unchartered territory. New physics is likely to show up and discovery will be followed by a phase of solution finding and optimization in view of the following steps (DEMO and alike.). This will be performed with the essential help of the other contributing facilities (figures 10 and 11).

On the technology side, the new burning plasma facilities also imply a huge step forward. They will be licensed as nuclear devices greatly extending the nuclear capabilities already demonstrated on JET and TFTR (tritium cycle, remote handling, shielding, etc). The question of the tritium inventory and its recovery stands as a major problem to solve and may require the use of different plasma facing materials such as tungsten.

If the objectives of the coming ICF and MCF burning plasma machines are the same in terms of power gain, they are vastly different in terms of fusion energy released per pulse: 20 to $40 \mathrm{MJ}$ for NIF and LMJ and $40000 \mathrm{MJ}$ for ITER. This means that ITER has the additional burden to actively cool all in-vessel components. Experience obtained with Tore Supra, TRIAM and LHD shows that this objective is a large qualitative step forward and the author expects that long pulse operation will be the object of a lengthy optimization in ITER. This phase is likely to be much more difficult to complete than obtaining the $Q=10$ objective. For ICF similarly, I expect that the question of producing low cost targets and high efficiency laser pulses at a rate of $10 \mathrm{~Hz}$ will be a major hurdle. The first successful fusion machine will be the one that produces in a reliable way a large amount of joules, not just watts! In this regard both MCF and ICF have a long way to go, even though MCF is a decade or two ahead of ICF.

Major tasks of a scientific and managerial nature are therefore ahead of us. A common observation is that the physicists and engineers necessary for these tasks are in rare supply. Clearly, it is important to strengthen the education channels capable of staffing the required task forces with highly qualified people.

More generally, it can be observed that the resources allocated to energy oriented fusion research are not commensurate with the stake and with the size of the tasks to be accomplished as a matter of urgency. Europe is considering a fast track for magnetic fusion. This is certainly, in the light of the challenges to be overcome, amply justified. The fast track should imply more resources given in priority to items on the critical path towards the realization of fusion energy. 


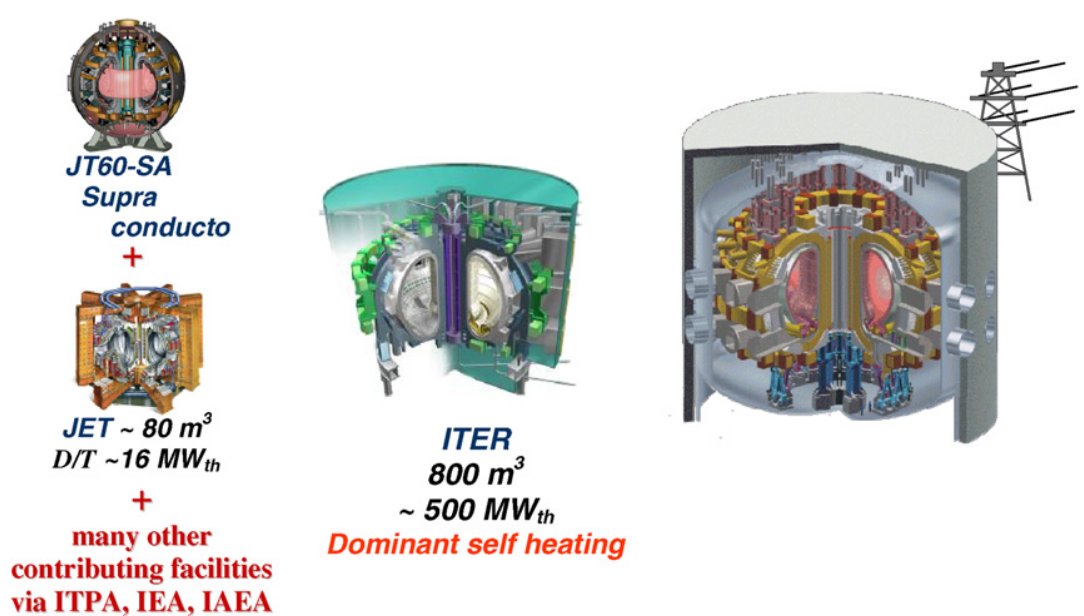

Figure 10. The way forward in magnetic fusion from existing experiments to DEMO on the right.

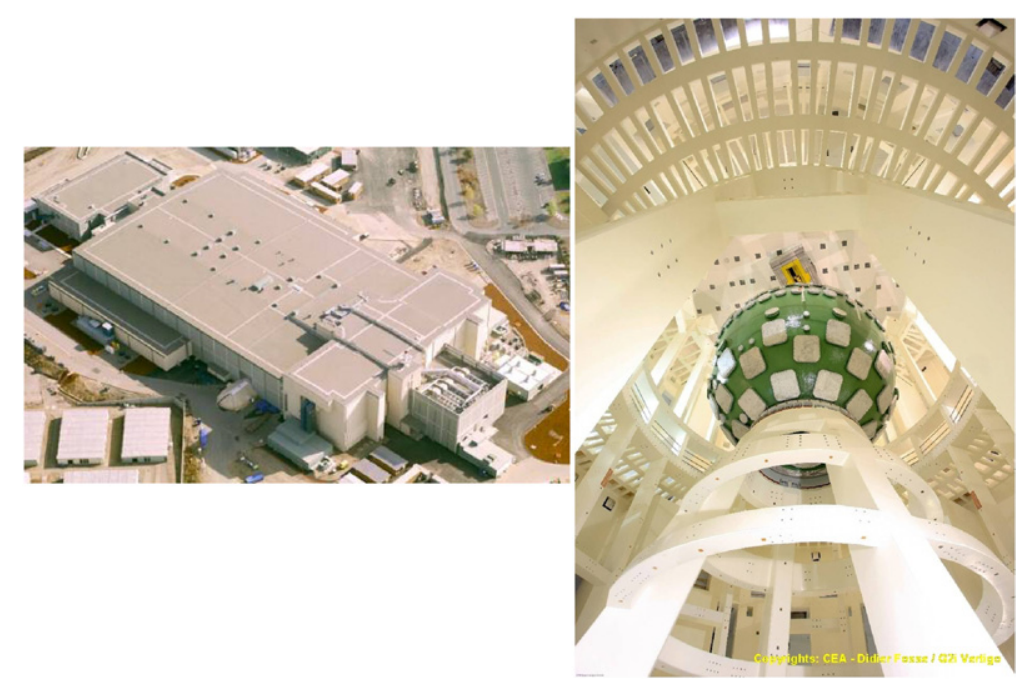

Figure 11. Two ignition $(Q \sim 10)$ facilities close to completion. Left: NIF in Livermore, USA and right: the experimental hall of LMJ in CESTA France.

This would be best accomplished by accelerating the ITER programme and putting increased emphasis on CW energy generation in ITER with long pulse or repetitive operation.

\section{Conclusions}

The legacy of 50 years in fusion can be summarized by a number of striking advances:

- a solid physics and technical basis for fusion has been constructed. Plasma science, not in existent in 1958, can now provide the tools for documenting plasma heating to 100 million degrees and confinement with thermonuclear characteristics. New technologies, for instance in the domains of superconductors and lasers, are now available for building the facilities capable of demonstrating burning plasmas for the first time in the history of mankind.

- A culture and a practice of international collaboration of unprecedented size and quality has been developed. It constitutes a major asset for meeting the formidable challenges lying ahead of us.
These challenges for the coming decades can also be illustrated by a few key items:

- scientists will explore the unchartered territory of the burning plasma states. Surprises, good and bad, are likely and will require a phase of solution finding and optimization.

- The construction of the new facilities has now moved into an area of a much more industrial nature. Fusion machines look terribly complicated in the eyes of industrialists. Several key items are the first of a kind! Scientists should endeavour to find ways of making the machine simpler. This is essential to contain costs and ensure reliability. The availability of a competent industry for highly specialized supplies is a challenge in its own. Fusion orders will have to compete with the renaissance of fission industry which is working at full capacity and hiring many qualified engineers. A novel modus vivendi with industry for coping with this situation is mandatory.

Fusion has ahead an exciting $Q=10$ burning plasma phase. Since 1958, it has withstood major difficulties. It is well armed 
to meet the challenges of the future. Even when the task seems impossible, the fusion community will have to remember its past which showed that

'when there is a (good) will, there is a way'.

\section{Acknowledgments}

It is a pleasure to thank the following colleagues for their kind help during the preparation of this paper: R. Arnoux, H. Azechi, B. Bigot; D. Besnard, S. Bourmaud, J. Ebrardt, M. Kikuchi, R. Stambaugh, G. Mank, A. Malaquias, D. Meade, E. Oktay, M. Skoric, Q. Tran and H. Yamada.

\section{References}

[1] Oliphant M.L.E., Harteck P. and Rutherford L. 1934 Transmutation effects observed with heavy hydrogen Proc R. Soc. A 144692

[2] Edited and introduced by Hendry J. 1984 Cambridge Physics in the 30's (Bristol: Hilger)

[3] Bethe H. and Peierls R. 1935 Quantum theory of the diplon Proc. R. Soc. Lond. A 148 146-56

[4] Lawson J.D. 1957 Some criteria for a power producing thermonuclear reactor Proc. Phys. Soc. B 70 6-10

[5] Tuck J.L. 1958 Review of Controlled Thermonuclear Research at Los Alamos for mid 1958 Proc. 1st Int. Conf. on Peaceful Uses of Atomic Energy (Geneva, 1958) vol 32 P/1860
[6] Thoneman P.C. 1958 Controlled Thermonuclear Research in the United Kingdom Proc. 1st Int. Conf. on Peaceful Uses of Atomic Energy (Geneva, 1958) vol 31 P/78

[7] Equipe T.F.R. 1980 Proc. IAEA FEC (Brussels, Belgium, 1980) vol II pp 552-3

[8] Galeev A., Sagdeev R., Galeev A.A. and Sagdeev R.Z. 1967 Zh. Eksp. Teor. Fiz. 53348

see also Galeev A., Sagdeev R., Galeev A.A. and Sagdeev R.Z. 1976 Advances in Plasma Physics vol 6 (New York: Wiley) pp 311-420 (A76-38891 19-75)

[9] Bickerton R.J., Connor J.W. and Taylor J.B. 1971 Nature Phys. Sci. 229110

[10] Jacquinot J., Keilhacker M. and Rebut P. 2008 Mission and highlights of the JET joint undertaking Fusion Sci. Technol. 53 866-90

[11] Goldston R. 1984 Plasma Phys. Control. Nucl. Fusion 2687

[12] Wagner F. et al 1982 Phys. Rev. Lett. 491408

[13] Troyon F., Gruber T., Sauremann H., Semazato S. and Succi S. 1984 MHD limited to plasma confinement Proc. 12th European Conf. on Plasma Physics and Controlled Fusion (Aachen, Germany, September 1983) vol 26 IA (Petit-Lancy: European Physical Society) p 209

[14] Greenwald M. et al 1988 Nucl. Fusion 282199

[15] Nuckolls J. et al 1972 Nature 239129

[16] Tabak M. et al 1994 Phys. Plasmas 11626

[17] Progress in the ITER Physics Basis 2007 Nucl. Fusion 47 $1-413$

[18] ten Kate H. 2008 ATLAS superconducting toroids completion, lessons learnt and how they can be used for fusion toroids Invited Lecture SOFT Rostock http://www.ipp.mpg.de/eng/ for/veranstaltungen/soft2008/ 